\title{
Antioxidation improves in puberty in normal weight and obese boys, in positive association with exercise-stimulated growth hormone secretion
}

\author{
George Paltoglou 1,2, loannis G. Fatouros ${ }^{3}$, George Valsamakis ${ }^{2}$, Maria Schoina ${ }^{3}$, Alexandra Avloniti ${ }^{3}$, Athanasios Chatzinikolaou ${ }^{3}$, \\ Antonis Kambas ${ }^{3}$, Dimitris Draganidis ${ }^{3}$, Aimilia Mantzou ${ }^{4}$, Maria Papagianni ${ }^{5}$, Christina Kanaka-Gantenbein ${ }^{2}$, George P. Chrousos ${ }^{2}$ \\ and George Mastorakos ${ }^{1}$
}

BACKGROUND: Oxidative stress is associated with obesity while the evidence for the role of $\mathrm{GH}$ in pro- and antioxidation is inconclusive. This study investigates the relationships between growth hormone $(\mathrm{GH})$, pro- and antioxidation in relation to obesity and puberty before and after an acute bout of exercise. METHODS: In this case-control study, 76 healthy normalweight and obese, prepubertal and pubertal boys underwent a blood sampling before and immediately after an aerobic exercise bout until exhaustion at 70\% maximal oxygen consumption. Markers of prooxidation (thiobarbituric acid reactive substances (TBARS) and protein carbonyls (PCs)) and antioxidation (glutathione (GSH), oxidized glutathione disulfide (GSSG), GSH/GSSG ratio, glutathione peroxidase (GPX), catalase, and total antioxidant capacity (TAC)) and hormones (GH, insulinlike growth factor (IGF)-1, IGF-BP-3, luteinizing hormone, follicle-stimulating hormone, and testosterone) were measured.

RESULTS: Baseline and postexercise TBARS and PCs were greater, while baseline GSH, GSH/GSSG ratio, GPX, and TAC were lower in obese than that in normal-weight participants. In all participants, waist was the best negative and positive predictor for postexercise GPX and TBARS, respectively. Baseline TAC was greater in pubertal than that in pre-pubertal participants. In all participants, baseline GH was the best negative predictor for postexercise PCs. Significant positive linear correlation exists between the exercise-associated GH, and GSSG increases in pubertal normal-weight boys.

CONCLUSIONS: Higher prooxidation and lower antioxidation were observed in obese boys, while antioxidation improves with puberty and postexercise, paralleling $\mathrm{GH}$ accentuated secretion.

O xidative stress defines a state of imbalance between proand antioxidation within the cell (1). Prooxidation refers to mitochondrial and nonmitochondrial mechanisms, which generate reactive oxygen and nitrogen species (RONS), whereas antioxidation refers to the adaptive activation of enzymatic and/ or nonenzymatic mechanisms, which scavenge prooxidants and their products within cells and in extracellular body fluids (1). Because the direct measurement of RONS is difficult to perform, thiobarbituric acid reactive substances (TBARS) and protein carbonyls (PCs) have been employed as markers of prooxidation (1). Glutathione (GSH) and oxidized glutathione disulfide (GSSG), the enzymes glutathione peroxidase (GPX) and catalase and the so-called total antioxidant capacity (TAC) have been employed as markers of antioxidation (2-4). Oxidative stress in humans has been associated with obesity and resulting comorbidities $(1,5,6)$. Childhood obesity has been associated with oxidative stress even before comorbidities occur $(6,7)$.

Puberty is a maturation period in human development, when sexual characteristics and reproductive competence are developed (8). It is characterized by changes in the dynamically regulated hypothalamic-growth hormone $(\mathrm{GH})$-insulin-like growth factor (IGF)-1 and hypothalamic-pituitary-gonadal axes (9). Children with GH deficiency demonstrate decreased production of antioxidation markers, which increase upon GH replacement therapy (10). In addition, in adults with GH deficiency, endothelial dysfunction is common and associated with the increased levels of prooxidation markers, which decrease following GH replacement therapy (11). Furthermore, physiological concentrations of testosterone have been shown in vitro to suppress neutrophil prooxidative capability (12). Also, the increasing testosterone levels during puberty in young Japanese boys correlated negatively with prooxidation markers (13). Previous studies have suggested that antioxidation in humans matures with age $(14,15)$.

Exercise represents a potent stimulus of energy substrate utilization in the mitochondrion and the cytosol that leads to the activation of prooxidation and the recruitment of antioxidation mechanisms (1). Moreover, exercise intensely stimulates the $\mathrm{GH}$ axis, and this stimulation is potentiated along puberty (16-18).

'Endocrine Unit, Aretaieion Hospital, University of Athens Medical School, Athens, Greece; 'First Department of Pediatrics, "Aghia Sofia" Children's Hospital, University of Athens Medical School, Athens, Greece; ${ }^{3}$ School of Physical Education and Sports Sciences, Democritus University of Thrace, Komotini, Greece; ${ }^{4}$ Endocrine Unit, Evgenidion Hospital, University of Athens Medical School, Athens, Greece; ${ }^{5}$ Pediatric Endocrinology Unit, Third Department of Pediatrics, Hippokrateion General Hospital of Thessaloniki, Aristotle University of Thessaloniki, Thessaloniki, Greece. Correspondence: George Mastorakos (mastorakg@ath.forthnet.gr) 
To investigate the relationships between GH, pro- and antioxidation in relation to obesity and puberty before and after an acute bout of exercise, 76 pre- and early pubertal normalweight and obese boys were studied at baseline and after an acute bout of aerobic exercise at $70 \%$ of maximal oxygen consumption $\left(\mathrm{VO}_{2} \max \right)$. Markers of the hormonal axes and of pro- and antioxidation were measured.

\section{RESULTS}

Baseline and Postexercise Concentrations of Pro- and Antioxidation Markers and Hormones

The baseline and postexercise concentrations (mean $\pm \mathrm{SE}$ ) of the prooxidation (TBARS, PCs) and the antioxidation (TAC, catalase, GPX, GSH, GSSG, and GSH/GSSG ratio) markers in the four groups of subjects studied, as well as their statistical comparisons, are reported in Figures 1 and 2. The baseline and postexercise concentrations of GH and IGF1 in the four groups of subjects studied, as well as their statistical comparisons, are reported in Figure 3.

The baseline concentrations of hemoglobin, hematocrit, IGFBP3, IGF1 to IGFBP3 ratio, luteinizing hormone (LH), follicle-stimulating hormone (FSH), and testosterone in the four groups of subjects studied, as well as their statistical comparisons, are reported in Table 1.

\section{Within-Group Correlations Between Concentrations of Pro- and Antioxidation Markers}

No statistically significant correlation was found between baseline concentrations of pro- and antioxidation markers in prepubertal normal-weight and obese subjects.
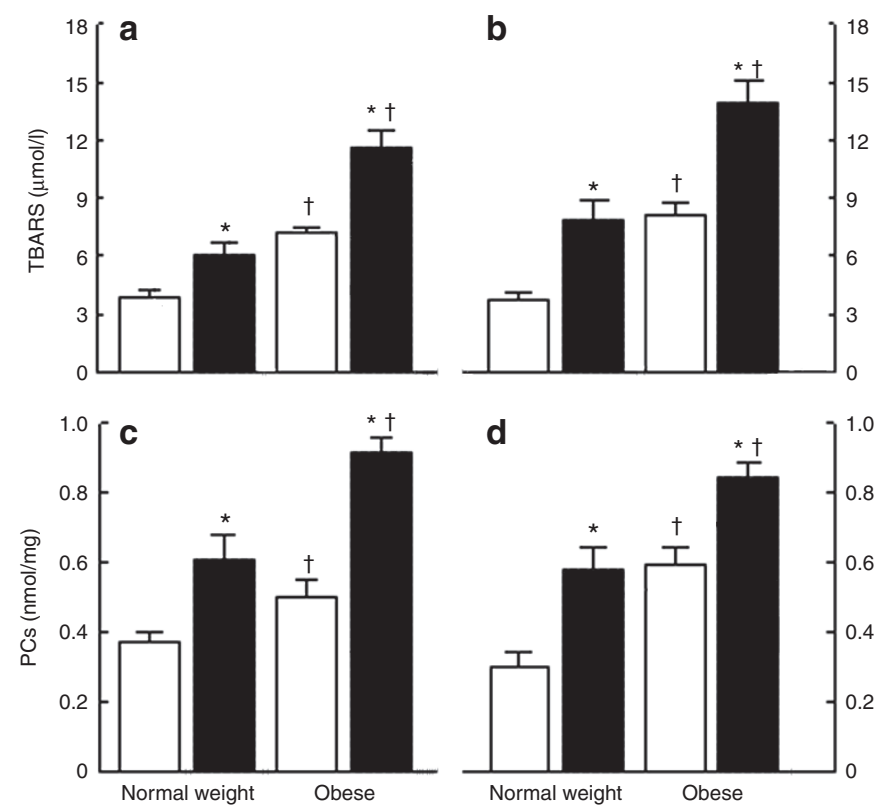

Figure 1. Markers of Pro-oxidation. Concentrations of thiobarbituric acid reactive substances (TBARS) and protein carobonyls (PCs) (mean \pm SE) at baseline (white bars) and postexercise (black bars) in (a,c) prepupertal and $(\mathbf{b}, \mathbf{d})$ pubertal normal-weight and obese subjects. *Significant difference $(P<0.05)$ from the respective baseline concentrations; ${ }^{\dagger}$ significant difference $(P<0.05)$ from normal-weight subjects.
In early pubertal normal-weight subjects, at baseline, TBARS and PCs concentrations correlated negatively with, respectively, TAC $(P<0.05 ; R=-0.94)$ and GSH $(P<0.05 ; R$ $=-0.94)$ concentrations. In early pubertal obese subjects, at baseline, PC concentrations correlated negatively with TAC $(P$ $<0.05 ; R=-0.76)$ and GSH $(P<0.05 ; R=-0.71)$ concentrations, as well as with GSH to GSSG ratio $(P<0.05 ; R=-0.68)$.

In prepubertal normal-weight subjects, $\triangle$ TBARS correlated positively with $\triangle \mathrm{TAC}(P<0.05 ; R=0.80)$. In early pubertal normal-weight subjects, $\triangle$ TBARS and $\triangle \mathrm{PCs}$ correlated positively with, respectively, $\Delta$ catalase $(P<0.05 ; R=0.98)$ and $\triangle$ GSSG $(P<0.05 ; R=0.95)$. In prepubertal obese subjects, $\triangle$ TBARS correlated positively with $\Delta \mathrm{GSH}(P<0.05 ; R=0.73)$.

\section{Within-Group Correlations Between GH, IGF1 and IGFBP3 Concentrations and IGF1 to IGFBP3 Ratio and Concentrations of Pro- and Antioxidation Markers}

In prepubertal normal-weight subjects, at baseline, $\mathrm{GH}$ concentrations correlated positively with TAC concentrations $(P<$ $0.05 ; R=0.68)$; IGF1 concentrations and IGF1 to IGFBP3 ratio correlated positively with catalase concentrations $(P<0.05 ; R$ $=0.62$ and $P<0.05 ; R=0.72$, respectively). In early pubertal normal-weight and obese subjects, at baseline, no statistically significant correlation was found among GH, IGF1, IGFBP3 concentrations and IGF1 to IGFBP3 ratio and concentrations of prooxidation or antioxidation markers.

In early pubertal normal-weight subjects, $\Delta \mathrm{GH}$ correlated positively with $\Delta$ GSSG $(P<0.05 ; R=0.94)$ (Figure 4). No statistically significant correlation was found among $\Delta$ hormonal concentrations, $\Delta$ pro oxidation and $\Delta$ antioxidation markers in prepubertal normal-weight and obese subjects and in early pubertal obese subjects.

\section{Correlations and Multiple Regression Analysis of All Subjects}

In all subjects taken as a single group, baseline TBARS correlated negatively with baseline TAC, GSH, GSH/GSSG, and GPX $(P<0.05 ; R=-0.63 ; R=-0.50, R=-0.43$, and $R=-0.46$, respectively). In all subjects taken as a single group, baseline PCs correlated negatively with baseline GSH, GSH/GSSG, and GPX $(P<0.05 ; R=-0.57, R=-0.52$, and $R=-0.67$, respectively). In all subjects taken as a single group, $\triangle \mathrm{PCs}$ correlated positively with $\triangle$ GSSG $(P<0.05 ; R=0.41)$. In all subjects taken as a single group, baseline $\mathrm{GH}$ correlated negatively with baseline PCs $(P<0.05 ; R=-0.46)$, while $\Delta \mathrm{GH}$ correlated positively with $\triangle$ GSSG $(P<0.05 ; R=0.53)$. Multiple regression analysis of all subjects taken as a single group revealed a statistically significant positive linear regression between the exercise-associated increase of $\mathrm{GH}(\Delta \mathrm{GH})$ and GSSG $(\Delta \mathrm{GSSG})(P<0.05 ; R=0.36)$.

Multiple regression analysis of all subjects taken as a single group revealed a statistically significant positive linear regression between the exercise-associated increase of $\mathrm{GH}(\Delta \mathrm{GH})$ and GSSG $(\triangle \mathrm{GSSG})(P<0.05 ; R=0.36)$.

\section{Predictors}

In all subjects taken as a single group, forward stepwise regression analysis was employed to reveal potential predictors of 

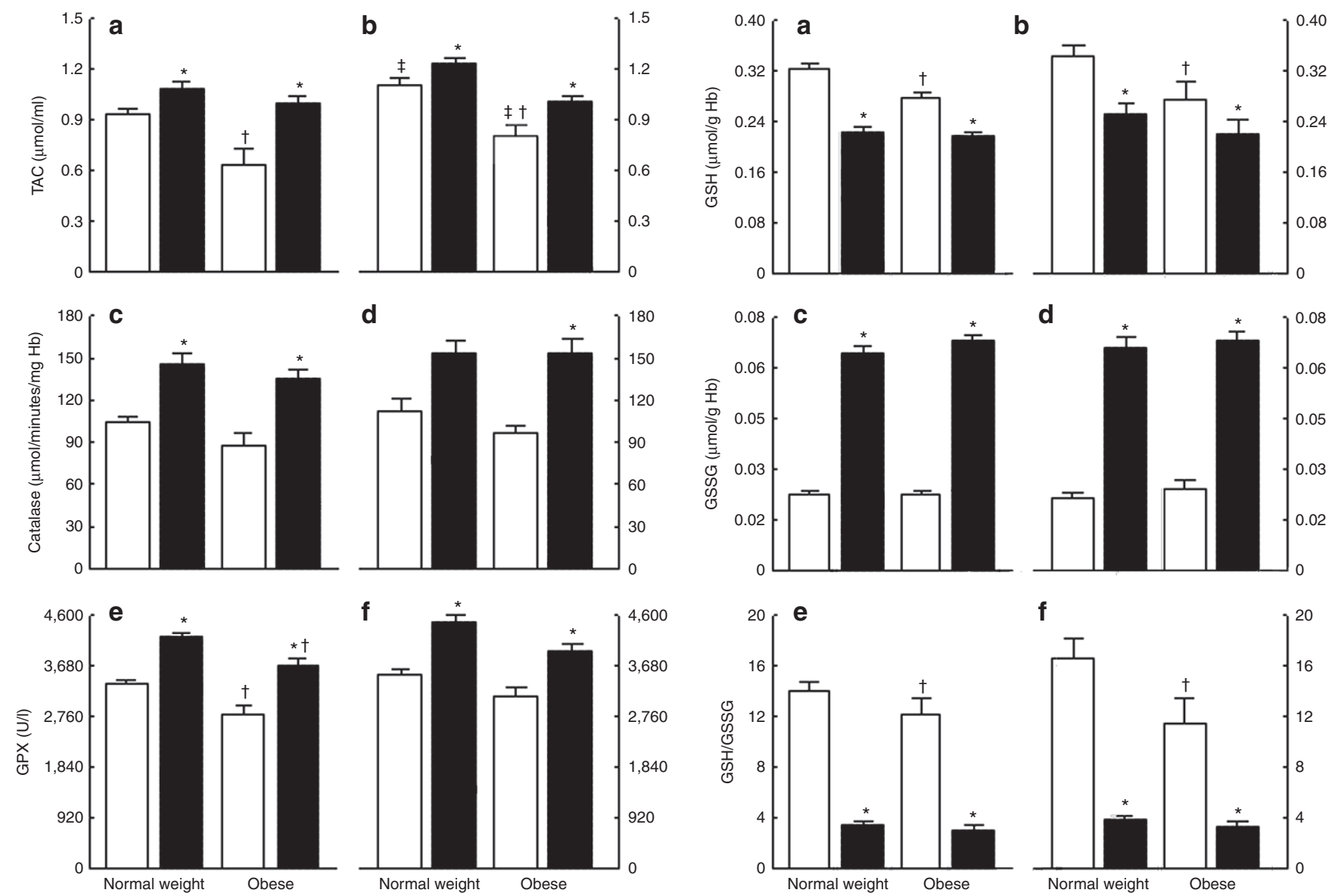

Figure 2. Markers of Anti-oxidation. Left side: (a,b) total antioxidant capacity (TAC), (c,d) catalase and (e,f) glutathione peroxidase (GPX) activity values (mean \pm SE) at baseline (white bars) and postexercise (black bars) in prepubertal (panels $\mathbf{a}, \mathbf{c}$, and e, respectively) and early pubertal (panels $\mathbf{b}$, $\mathbf{d}$, and $\mathbf{f}$, respectively) normal-weight and obese subjects. Right side: (a,b) glutathione (GSH), (c,d) oxidized glutathione (GSSG) concentrations and (e,f) glutathione to oxidized glutathione ratio (GSH/GSSG (mean \pm SE)) at baseline (white bars) and postexercise (black bars) in prepubertal (panels a, $\mathbf{c}$, and $\mathbf{e}$, respectively) and early pubertal (panels $\mathbf{b}$, $\mathbf{d}$, and $\mathbf{f}$, respectively) normal-weight and obese subjects. *Significant difference $(P<0.05)$ from the respective baseline concentrations; ${ }^{\dagger}$ significant difference $(P<0.05)$ from normal-weight subjects; ${ }^{\ddagger}$ significant difference $(P<0.05)$ from prepubertal subjects.

the postexercise concentrations of the prooxidation (PCs and TBARS) and the antioxidation (GSH, GSSG, GSH/GSSG ratio, catalase, and TAC) markers, each one taken as dependent variable, among baseline waist circumference, $\mathrm{VO}_{2} \max , \mathrm{GH}$, IGF1/IGFBP3 ratio, LH, and testosterone, all taken as independent variables. Baseline $\mathrm{GH}$ was the best negative predictor $(P<0.05 ; b=-0.37)$ for postexercise concentrations of PCs, while baseline waist circumference was the best negative and positive predictor for postexercise concentrations of GPX $(P<$ $0.05 ; b=-0.72)$ and TBARS $(P<0.05 ; \mathrm{b}=0.74)$, respectively.

In all subjects taken as a single group, forward stepwise regression analysis was employed to reveal potential predictors of the change $\Delta$ of the prooxidation (PCs and TBARS) and the antioxidation (GSH, GSSG, GSH/GSSG ratio, catalase, and TAC) markers, each one taken as a dependent variable, among baseline waist circumference, $\mathrm{VO}_{2} \max , \mathrm{GH}$, IGF1/ IGFBP3, LH, and testosterone, all taken as independent variables. Baseline waist circumference was the best negative predictor for the absolute change of GSH $(\triangle \mathrm{GSH})(P<0.05 ; b=$ $-0.48)$ and the best positive predictor for $\triangle \mathrm{TBARS}(P<0.05$; $\mathrm{b}=0.64)$.

\section{DISCUSSION}

We found that in pre- and early puberty, baseline concentrations of pro- and antioxidation markers were significantly greater and lower, respectively, in obese than that in normal-weight subjects. In early puberty, baseline antioxidation markers correlated negatively with prooxidation markers both in the obese and normal-weight subjects. Increased baseline prooxidation (or reduced antioxidation) markers in obese pre- and early pubertal subjects have been reported (6). Also, BMI in children and adolescents has been correlated positively and negatively, respectively, with pro- and antioxidation markers (19). The imbalance between pro- and antioxidation mechanisms observed in obesity might contribute to the pathogenesis of obesity and metabolic syndrome-associated comorbidities (6). Of note, adipose tissue-related aseptic low-grade inflammation might contribute to reduced antioxidant capacity and increase of RONS production $(1,5)$. In addition, in this study, the baseline concentrations of TAC, a marker of antioxidation, were greater in early than that in prepubertal normal-weight and obese subjects. This finding could imply that antioxidation mechanisms might become more efficient with the progress of puberty. However, 
to confirm this, studies of a wider age-range population, spanning over all stages of puberty, should be undertaken $(14,15)$. The suggested maturation of antioxidation mechanisms along
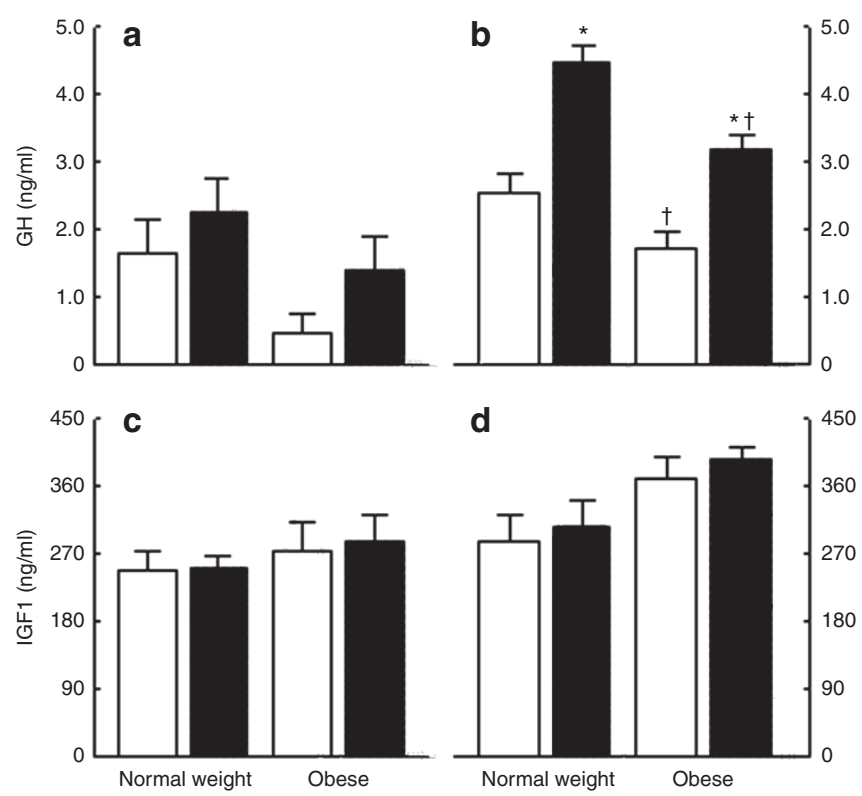

Figure 3. Growth Hormone - IGF1 axis. (a,b) Growth hormone (GH) and (c,d) insulin-like growth factor (IGF)-1 concentrations (mean \pm SE) at baseline (white bars) and postexercise (black bars) in prepubertal $(\mathbf{a}, \mathbf{c})$ and early pubertal $(\mathbf{b}, \mathbf{d})$ normal-weight and obese subjects. ${ }^{*}$ Significant difference $(P<$ 0.05 ) of postexercise from the respective baseline concentrations; ${ }^{\dagger}$ significant difference $(P<0.05)$ between obese and respective normal-weight subjects. with lower production of RONS by the mitochondria of primates might explain why these species live longer than expected (15). Of note, in this study, baseline testosterone concentrations were greater in normal-weight than that in obese early pubertal subjects. Lower concentrations of total testosterone with normal testicular volumes and comparable LH and FSH concentrations have been described in obese boys, associated with the progression of puberty $(20,21)$. This phenomenon has been attributed to the reduced sex hormone-binding globulin (SHBG) binding capacity, the decreased LH pulse amplitude, and the relative increase of estrogen production (21).

In this study, following an acute bout of aerobic exercise, markers of both pro- and antioxidation mechanisms increased in all studied groups, while their changes correlate positively to each other, suggesting that the former are recruited to counteract the latter. Although the exercise duration till exhaustion was shorter in obese than that in normal-weight early pubertal boys, it did not seem to influence the activation of prooxidation and the recruitment of antioxidation mechanisms postexercise. Exercise increases oxygen consumption by muscle cells and stimulates energy substrate utilization in the mitochondrion and the cytosol that leads to generation of RONS (1). The latter are also produced following muscle injury, through generation by phagocytic white cells, muscle calcium accumulation, and through disruption of iron-containing proteins (1). In the past, an acute bout of vigorous exercise in healthy children initiated a catabolic hormonal response and cytokine-mediated response which is followed by recruitment of scavenging antioxidation mechanisms, such as GSH, leading

Table 1. Anthropometric and exercise data measurements, hemoglobin, hematocrit, IGFBP3, IGF1 to IGFBP3 ratio, LH, FSH, and testosterone baseline concentrations in normal-weight and obese, prepubertal and early pubertal subjects

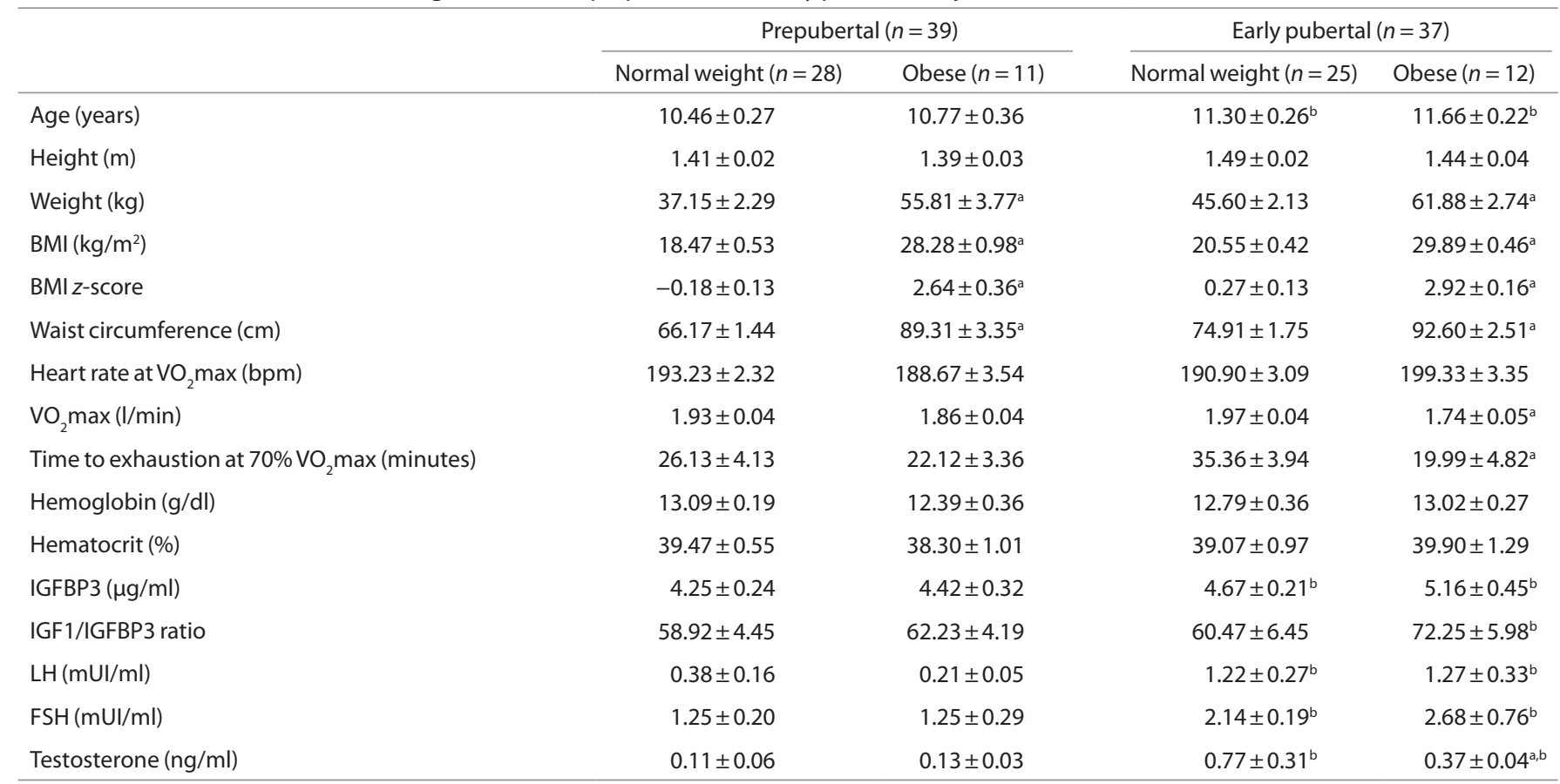

Measurements were compared with Factors ANOVA followed by LSD Fischer's post hoc test $(P<0.05)$.

FSH, follicle-stimulating hormone; IGF, insulin-like growth factor; LH, luteinizing hormone; VO2max, maximal oxygen consumption.

aSignificant difference $(P<0.05)$ between obese and respective normal weight subjects. ${ }^{\circ}$ Significant difference $(P<0.05)$ between early pubertal and respective prepubertal subjects. 


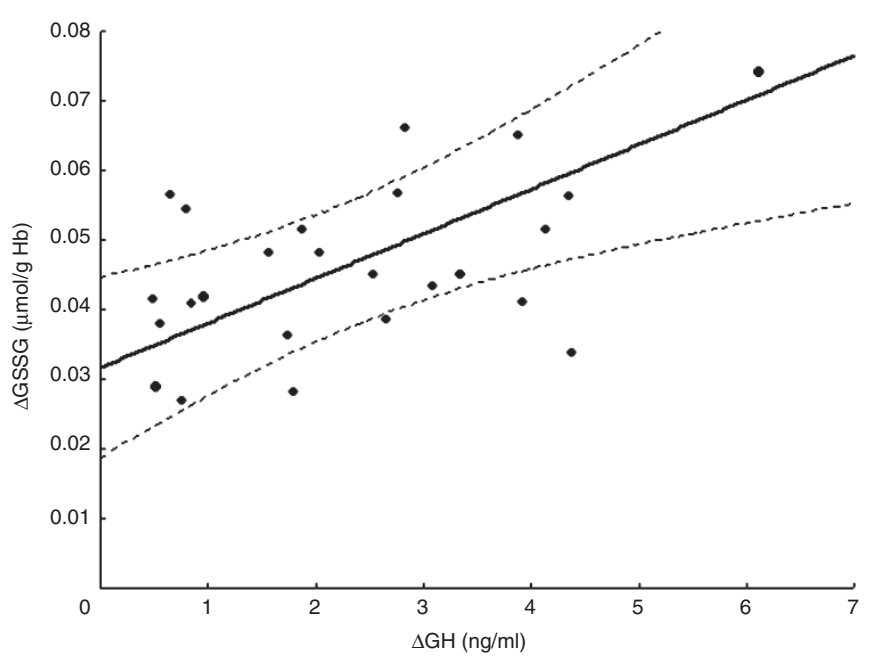

Figure 4. Statistically significant linear correlation in early pubertal normal-weight subjects between $\Delta \mathrm{GH}$ and $\Delta \mathrm{GSSG}(P<0.05 ; r=0.94)$. GH, growth hormone; GSSG, oxidized glutathione.

to limitation of the destruction (22). In prepuberty, exerciseassociated increase of prooxidation markers was greater in obese than that in normal-weight subjects. Also, in pre- and early puberty, exercise-associated increase of antioxidative GPX activity was lower in obese than that in normal-weight subjects. When all subjects were taken as one group, among all baseline variables employed, waist circumference was the best negative predictor for postexercise GPX activity, as well as for $\triangle \mathrm{GSH}$, and the best positive predictor for postexercise, prooxidation TBARS and $\triangle$ TBARS.

In this study, GH concentrations were lower in obese than that in normal-weight early pubertal subjects in accordance with previous reports regarding basal and stimulated GH secretion (23). In obesity, GH secretion may be inhibited at the level of pituitary and hypothalamus by increased insulin, free IGF1, and free fatty acid concentrations (24). In this study, GH concentrations increased following exercise in all groups, but significant increase was observed only in early pubertal subjects, indicating a more eager exercise-stimulated GH secretion in puberty, confirming that exercise-induced GH increase parallels puberty progression $(16,25)$. Exercise represents a stimulus of GH secretion, probably through the JAK2-STAT5 phosphorylation pathway $(17,18,26,27)$. IGF1 and IGFBP3 concentrations did not change following the acute exercise bout, as previously described (25). IGF1 and IGFBP3, while mediating some of the tissue-specific $\mathrm{GH}$ effects, seem to require longer exercise bouts or chronic training to be increased (28). In the present study, baseline GH, IGF1, and IGF1/IGFBP3 ratio correlated positively with antioxidation markers in prepubertal normal-weight subjects. Of note, GSH is produced by the liver, a target-organ of GH action, where it upregulates IGF1 production (29). When all subjects were taken as one group, baseline $\mathrm{GH}$ was the best negative predictor for postexercise PCs, a marker of prooxidation, while exerciseassociated increase of GH concentrations correlated positively with the exercise-associated GSSG increase, an antioxidation marker, and negatively with PCs, a prooxidation marker.
Thus, the increase of $\mathrm{GH}$ is associated with that of antioxidation products in agreement with previously described antioxidation effects of GH (10). In humans, STAT 5B phosphorylation appears to be a very robust marker of intracellular $\mathrm{GH}$ signaling in vivo (27). The promoter of the GSH synthetase gene contains multiple binding sites for STAT family members including STAT5, suggesting that STAT5 might regulate the transcriptional activation of this gene (30). Interestingly, a threefold increase of RONS amounts was observed in STAT5A knockout cells as compared with STAT5A-expressing cells (30). Moreover, the synthesis of GSH or the activities of antioxidant enzymes such as GSH reductase and GPX are modulated by the Hsp27 and Hsp70 that are likely to be also modulated by STAT5 expression (31). Furthermore, Fukushima et al. (32) have recently shown that $6 \mathrm{wk}$ of GH treatment of obese mice resulted to suppressed TBARS expression in the epididymal fat and enhanced antioxidant enzyme gene expression. Indeed, children with GH deficiency and decreased IGF1 concentrations demonstrate substantially increased oxidative stress that decreases after GH replacement therapy (10). Furthermore, in $\mathrm{GH}$-deficient adults, the presence of endothelial dysfunction is associated with enhanced oxidative stress that diminishes following GH replacement (11).

In summary, following an acute bout of aerobic exercise, both pro- and antioxidation mechanisms are stimulated in normal-weight and obese, pre- and early pubertal boys. The antioxidant capacity of the organism improves with the onset of puberty. This might be related to the finding that GH is positively associated with antioxidation at baseline and postexercise. Obese subjects demonstrate greater and lower pro- and antioxidation mechanisms, respectively, than normal-weight subjects, while also demonstrating lower GH concentrations. These observations provide on one hand a conceptual link between early pubertal obesity and increased prooxidation, highlighting the deleterious potential of obesity, while on the other hand, they suggest the implication of pubertal physiological mechanisms in the maturation of antioxidation.

In conclusion, moderate acute aerobic exercise is a good model for the study of pro- and antioxidation mechanisms in children and adolescents. The suggested maturation of the antioxidation mechanisms during the transition to early puberty in humans should be studied further. Therefore, studies in the future need to investigate the interplay between exercise, onset of puberty, and energy consumption and storage, especially regarding the increasing prevalence of obesity in the pediatric population.

\section{METHODS}

\section{Subjects}

Male students (117 in total) in the fifth and sixth grades in Komotini, Greece (aged $10.95 \pm 0.99$ y (mean \pm SD)) were initially enrolled in this study. After application of the following exclusion criteria, such as (i) exercise additional to that included in the school time-table, (ii) nutritional intervention within the 6 mo preceding this study, and (iii) history of diabetes, insulin resistance, dyslipidemia, cardiovascular disease, and hypertension or other known chronic pathology, their BMI was calculated and compared with the standard BMI curves for the Greek pediatric population, according to the International Obesity 
Task Force criteria $(33,34)$. Only healthy normal-weight $(N=53)$ and obese $(N=23)$ boys from the examined school classes were included in this study. Subjects were considered normal-weight or obese when their projected BMI value for the age of $18 \mathrm{y}$ was lower than $25 \mathrm{~kg}$ / $\mathrm{m}^{2}$ or between 30 and $35 \mathrm{~kg} / \mathrm{m}^{2}$, respectively (34). Of the 117 originally recruited boys, 23 were obese, representing a ratio $19.66 \%$ that approximates the ratio $13.6 \%$ according to the International Obesity Task Force criteria for obesity in this age group for the respective part of Greece (35). In addition, subjects with testosterone concentration greater than $0.2 \mathrm{ng} / \mathrm{ml}$ were considered as early pubertal $(36,37)$. Thus, from the 76 subjects, 39 were prepubertal (mean \pm SD; aged $10.59 \pm 0.22 \mathrm{y}$ ) and 37 were early pubertal (aged $11.44 \pm 0.18 \mathrm{y}$ ). Subsequently, four groups of subjects were formed: (i) prepubertal normal-weight $(N=28)$, (ii) prepubertal obese $(N=11)$, (iii) early pubertal normal-weight $(N=25)$, and (iv) early pubertal obese $(N=$ 12). Subjects' anthropometric characteristics are shown in Table 1.

\section{Protocol}

The study was approved by the Institutional Review Board (University of Athens, Medical School) and was conducted in accordance with the Declaration of Helsinki as revised in 1996. Informed written consent was obtained from the parent/guardians of each child while children gave verbal consent to participate in the study. The protocol was performed in two visits separated by $2 \mathrm{wk}$ in a university ergophysiology laboratory.

\section{First Visit (Subject Selection and Maximal Oxygen Consumption Measurement)}

During the first visit, all 117 boys, recruited after application of the exclusion criteria, were examined by the same experienced pediatrician (M.S.). Participants had their body weight measured to the nearest $0.1 \mathrm{~kg}$ (Beam Balance 710; Seca, Birmingham, UK) while they were wearing underclothes. Barefoot standing height was measured to the nearest $0.1 \mathrm{~cm}$ (Stadiometer 208; Seca, Hanover, MD), and their $\mathrm{BMI}$ and BMI $z$-scores were calculated (34). Maximum waist circumference was measured in duplicate using a flexible measuring tape. A blood sample was drawn from all normal-weight (53) and obese (23) boys for $\mathrm{LH}, \mathrm{FSH}$, and testosterone measurements.

Normal-weight and obese participants had their $\mathrm{VO}_{2}$ max measured, by performing a graded exercise test until maximum exercise tolerance on a stationary cycle ergometer (Monark 834E; Monark, Varberg, Sweden) according to a previously described protocol (38). Open-circuit spirometry via continuous breath-by-breath analysis (averaged every 30 s) was used to measure $\mathrm{VO}_{2}$ max with an automated online pulmonary gas exchange system (SensorMedics 2900c; SensorMedics Yorba Linda, CA). Heart rate, 12-lead electrocardiogram, blood pressure, and ratings of perceived exertion were monitored continuously throughout testing and during recovery. $\mathrm{VO}_{2}$ max was attained if: (i) subject reached exhaustion (a pedaling rate $<60$ revolutions/min), (ii) respiratory exchange ratio was $\geq 1.10$, (iii) a $\mathrm{VO}_{2}$ plateau was observed (increase of $<2 \mathrm{ml} /$ $\mathrm{kg} / \mathrm{min}$ ) despite further increases of the workload, and (iv) a heart rate was within $10 \mathrm{bpm}$ of the theoretical maximum heart rate (220-age). All participants met all four criteria. Subjects' heart rate at $\mathrm{VO}_{2} \max (\mathrm{bpm})$ and $\mathrm{VO}_{2} \max (1 / \mathrm{min})$ values are shown in Table 1.

\section{Second Visit (Baseline Sampling, Aerobic Exercise Bout, and Postexercise Sampling)}

Only normal-weight and obese participants had a second visit. The experiment was executed between 08:00 and 10:00 in the morning. A baseline blood sampling was performed followed by an acute bout of aerobic exercise on a stationary cycle ergometer (Monark 834E) until exhaustion (a pedaling rate $<60$ revolutions $/ \mathrm{min}$ ) at an intensity corresponding to $70 \%$ of their $\mathrm{VO}_{2}$ max. All participants completed this bout of exercise successfully. Immediately after exhaustion (end of the exercise bout), a second (post-exercise) blood sampling was performed. Subjects' time to exhaustion at $70 \% \mathrm{VO}_{2} \max (\mathrm{min})$ values are shown in Table 1.

\section{Assays}

Blood was collected from an antecubital vein (kept patent by flushing with normal saline) into EDTA tubes or tubes containing SSTGel (BD Vacutainer, NJ) and subsequently centrifuged for plasma or serum separation, respectively. Red blood cells collected after plasma separation were lysed, and the lysate was utilized for the analysis of
GSH, GSSG, and catalase activities $(2,3)$. Serum was utilized for hormonal analysis as well as measurement of TBARS, PCs concentration, and TAC (4). Whole blood was utilized for the measurement of hemoglobin, hematocrit, and GPX activity (2). Samples were stored frozen in multiple aliquots (serum and lysate samples at $-80^{\circ} \mathrm{C}$ and whole blood at $-20^{\circ} \mathrm{C}$ ), were thawed once before analysis, and were protected from light and autoxidation. All assays were performed in duplicate, and the mean value was recorded.

\section{Oxidative Stress Markers}

The measured prooxidation markers were TBARS and PCs. Serum TBARS and PCs were measured with a spectrophotometric method as previously described (4). The intra- and interassay coefficients of variation (CV) were 4.3 and $6.8 \%$, for TBARS, and 4.2 and $6.3 \%$ for PCs, respectively. The measured antioxidation markers were GSH and GSSG, the enzymes GPX and catalase as well as TAC. Erythrocyte lysate GSH and GSSG were measured with a spectrophotometric method as previously described (3). Serum TAC was measured with a spectrophotometric method as previously described (4).Erythrocyte lysate catalase activity was measured with a spectrophotometric method as previously described (3). Whole blood GPX was measured with a spectrophotometric method, with the use of a commercially available kit (Ransel RS 505; Randox, Crumlin, UK), as previously described (2). The intra- and interassay CV were 3.7 and $6.4 \%$ for GSH, 5.5 and $7.6 \%$ for GSSG, 3.8 and $5.4 \%$ for TAC, 5.9 and $7.9 \%$ for catalase activity, and 4.5 and $4.4 \%$ for GPX, respectively.

\section{Hormone Assays}

GH, IGF1, IGFBP3, FSH, LH, and testosterone were measured with a solid-phase, competitive chemiluminescent enzyme immunoassay (Immulite 2000; Siemens, Erlangen, Germany). The intra-assay $\mathrm{CV}$, the interassay $\mathrm{CV}$, and the assay sensitivity were $3.5 \%, 6.5 \%$, and $0.01 \mathrm{ng} / \mathrm{ml}$ for $\mathrm{GH} 2.9 \%, 7.4 \%$, and $20 \mathrm{ng} / \mathrm{ml}$ for IGF1, $4.2 \%$, $7.3 \%$, and $0.1 \mu \mathrm{g} / \mathrm{ml}$ for IGFBP3, $2.9 \%, 4.1 \%$, and $0.1 \mathrm{mIU} / \mathrm{ml}$ for FSH, 3.04\%, $6.6 \%$, and $0.05 \mathrm{mIU} / \mathrm{ml}$ for $\mathrm{LH}$, and $11.7 \%, 13.0 \%$, and $0.15 \mathrm{ng} / \mathrm{ml}$ for testosterone, respectively.

\section{Statistical Analyses}

Results are reported as means \pm SE. Statistical significance was set at $P<0.05$. All variables were normally distributed. All measured variables assayed at pre- (baseline) and postexercise were compared by employing repeated-measures ANOVA. All variables measured only at baseline were compared among groups by employing factors ANOVA. Significant main effects were revealed by the Fischer's LSD post hoc test. The Fischer's least significant differences post hoc test employed for the statistical analyses of this study compensates for unequal sample sizes. To reinforce the statistical robustness of the analyses performed, the Unequal N HSD post hoc test, that is better suited for unequal sample sizes, was also employed to reanalyze the data of the study wherever indicated. The results of the analyses with both statistical approaches were concordant. Within groups and for all subjects, correlations of baseline value and the absolute difference between pre- and postexercise values $(\Delta)$ were evaluated by the Pearson's R coefficient. A multiple regression analysis was performed for all subjects between $\Delta$ concentrations of hormones and pro- and antioxidation markers measured. To investigate potential predictors of the postexercise oxidation markers (dependent variables) among baseline waist circumference, $\mathrm{VO}_{2}$ max, GH, IGF1/IGFBP3, LH, and testosterone (independent variables), a standard forward stepwise regression model was employed for all subjects. All statistical evaluations were performed with the STATISTICA 6 software (STATSOFT, Tulsa, OK).

\section{STATEMENT OF FINANCIAL SUPPORT}

The authors did not receive any financial support.

Disclosure: The authors have nothing to disclose.

\section{REFERENCES}

1. Fisher-Wellman K, Bloomer RJ. Acute exercise and oxidative stress: a 30 year history. Dyn Med 2009;8:1.

2. Germanou EI, Chatzinikolaou A, Malliou P, et al. Oxidative stress and inflammatory responses following an acute bout of isokinetic exercise in obese women with knee osteoarthritis. Knee 2013;20:581-90. 
3. Margonis K, Fatouros IG, Jamurtas AZ, et al. Oxidative stress biomarkers responses to physical overtraining: implications for diagnosis. Free Radic Biol Med 2007;43:901-10.

4. Barbas I, Fatouros IG, Douroudos II, et al. Physiological and performance adaptations of elite Greco-Roman wrestlers during a one-day tournament. Eur J Appl Physiol 2011;111:1421-36.

5. Hopps E, Noto D, Caimi G, Averna MR. A novel component of the metabolic syndrome: the oxidative stress. Nutr Metab Cardiovasc Dis 2010;20:72-7.

6. Codoñer-Franch P, Tavárez-Alonso S, Murria-Estal R, Tortajada-Girbés M, Simó-Jordá R, Alonso-Iglesias E. Elevated advanced oxidation protein products (AOPPs) indicate metabolic risk in severely obese children. Nutr Metab Cardiovasc Dis 2012;22:237-43.

7. Atabek ME, Vatansev H, Erkul I. Oxidative stress in childhood obesity. J Pediatr Endocrinol Metab 2004;17:1063-8.

8. Burt Solorzano CM, McCartney CR. Obesity and the pubertal transition in girls and boys. Reproduction 2010;140:399-410.

9. Veldhuis JD, Roemmich JN, Richmond EJ, Bowers CY. Somatotropic and gonadotropic axes linkages in infancy, childhood, and the puberty-adult transition. Endocr Rev 2006;27:101-40.

10. Mohn A, Marzio D, Giannini C, Capanna R, Marcovecchio M, Chiarelli F. Alterations in the oxidant-antioxidant status in prepubertal children with growth hormone deficiency: effect of growth hormone replacement therapy. Clin Endocrinol (Oxf) 2005;63:537-42.

11. Evans LM, Davies JS, Anderson RA, et al. The effect of GH replacement therapy on endothelial function and oxidative stress in adult growth hormone deficiency. Eur J Endocrinol 2000;142:254-62.

12. Marin DP, Bolin AP, dos Santos Rde C, Curi R, Otton R. Testosterone suppresses oxidative stress in human neutrophils. Cell Biochem Funct 2010;28:394-402.

13. Kogawa T, Nishimura M, Kurauchi S, Kashiwakura I. Characteristics of reactive oxygen metabolites in serum of early teenagers in Japan. Environ Health Prev Med 2012;17:364-70.

14. Benitez-Sillero Jde D, Perez-Navero JL, Tasset I, Guillen-Del Castillo M, Gil-Campos M, Tunez I. Influence of intense exercise on saliva glutathione in prepubescent and pubescent boys. Eur J Appl Physiol 2009;106:181-6.

15. Finkel T, Holbrook NJ. Oxidants, oxidative stress and the biology of ageing. Nature 2000;408:239-47.

16. Bouix O, Brun JF, Fédou C, et al. Plasma beta-endorphin, corticotrophin and growth hormone responses to exercise in pubertal and prepubertal children. Horm Metab Res 1994;26:195-9.

17. Luger A, Watschinger B, Deuster P, Svoboda T, Clodi M, Chrousos GP. Plasma growth hormone and prolactin responses to graded levels of acute exercise and to a lactate infusion. Neuroendocrinology 1992;56:112-7.

18. Mastorakos G, Pavlatou M, Diamanti-Kandarakis E, Chrousos GP. Exercise and the stress system. Hormones (Athens) 2005;4:73-89.

19. Faienza MF, Francavilla R, Goffredo R, et al. Oxidative stress in obesity and metabolic syndrome in children and adolescents. Horm Res Paediatr 2012;78:158-64.

20. Mogri M, Dhindsa S, Quattrin T, Ghanim H, Dandona P. Testosterone concentrations in young pubertal and post-pubertal obese males. Clin Endocrinol (Oxf) 2013;78:593-9.

21. Vandewalle S, Taes Y, Fiers T, et al. Sex steroids in relation to sexual and skeletal maturation in obese male adolescents. J Clin Endocrinol Metab 2014;99:2977-85.
22. Pompella A, Visvikis A, Paolicchi A, De Tata V, Casini AF. The changing faces of glutathione, a cellular protagonist. Biochem Pharmacol 2003; 66:1499-503.

23. Ballerini MG, Ropelato MG, Domené HM, Pennisi P, Heinrich JJ, Jasper HG. Differential impact of simple childhood obesity on the components of the growth hormone-insulin-like growth factor (IGF)-IGF binding proteins axis. J Pediatr Endocrinol Metab 2004;17:749-57.

24. Romero CJ, Pine-Twaddell E, Sima DI, et al. Insulin-like growth factor 1 mediates negative feedback to somatotroph GH expression via POU1F1/ CREB binding protein interactions. Mol Cell Biol 2012;32:4258-69.

25. Pomerants T, Tillmann V, Karelson K, Jürimäe J, Jürimäe T. Impact of acute exercise on bone turnover and growth hormone/insulin-like growth factor axis in boys. J Sports Med Phys Fitness 2008;48:266-71.

26. Consitt LA, Wideman L, Hickey MS, Morrison RF. Phosphorylation of the JAK2-STAT5 pathway in response to acute aerobic exercise. Med Sci Sports Exerc 2008;40:1031-8.

27. Møller N, Jørgensen JO. Effects of growth hormone on glucose, lipid, and protein metabolism in human subjects. Endocr Rev 2009;30:152-77.

28. Gatti R, De Palo EF, Antonelli G, Spinella P. IGF-I/IGFBP system: metabolism outline and physical exercise. J Endocrinol Invest 2012;35:699-707.

29. Bianchi G, Bugianesi E, Ronchi M, Fabbri A, Zoli M, Marchesini G. Glutathione kinetics in normal man and in patients with liver cirrhosis. J Hepatol 1997;26:606-13.

30. Cholez E, Debuysscher V, Bourgeais J, et al. Evidence for a protective role of the STAT5 transcription factor against oxidative stress in human leukemic pre-B cells. Leukemia 2012;26:2390-7.

31. Guo S, Wharton W, Moseley P, Shi H. Heat shock protein 70 regulates cellular redox status by modulating glutathione-related enzyme activities. Cell Stress Chaperones 2007;12:245-54.

32. Fukushima M, Okamoto Y, Katsumata H, et al. Growth hormone ameliorates adipose dysfunction during oxidative stress and inflammation and improves glucose tolerance in obese mice. Horm Metab Res 2014;46: 656-62.

33. Chiotis D, Krikos X, Tsiftis G, Hatzisymeon M, Maniati-Christidi M, Dacou-Voutetaki A. Body mass index and prevalence of obesity in subjects of Hellenic origin aged 0-18 years living in the Athens area. Ann Clin Pediatr Unive Atheniensis 2004; 51:139-54.

34. Cole TJ, Bellizzi MC, Flegal KM, Dietz WH. Establishing a standard definition for child overweight and obesity worldwide: international survey. BMJ 2000;320:1240-3.

35. Mavrakanas TA, Konsoula G, Patsonis I, Merkouris BP. Childhood obesity and elevated blood pressure in a rural population of northern Greece. Rural Remote Health 2009;9:1150.

36. Veldhuis JD, Pincus SM, Mitamura R, et al. Developmentally delimited emergence of more orderly luteinizing hormone and testosterone secretion during late prepuberty in boys. J Clin Endocrinol Metab 2001;86:80-9.

37. Albertsson-Wikland K, Rosberg S, Lannering B, Dunkel L, Selstam G, Norjavaara E. Twenty-four-hour profiles of luteinizing hormone, folliclestimulating hormone, testosterone, and estradiol levels: a semilongitudinal study throughout puberty in healthy boys. J Clin Endocrinol Metab 1997;82:541-9.

38. Kelly AS, Steinberger J, Olson TP, Dengel DR. In the absence of weight loss, exercise training does not improve adipokines or oxidative stress in overweight children. Metabolism 2007;56:1005-9. 